\title{
IMPEDANCE AND INSTABILITIES IN THE NLC DAMPING RINGS
}

\author{
J. Corlett ${ }^{\dagger}$ D. Li, M. Pivi, R. Rimmer, S. DeSantis, A. Wolski, LBNL, Berkeley, CA, USA \\ A. Novokhatski, C. Ng, SLAC, Stanford, CA, USA
}

\begin{abstract}
We report on impedance calculations and single-bunch and multi-bunch instabilities in the NLC damping rings. Preliminary designs of vacuum chambers and major components have addressed beam impedance issues, with the desire to increase instability current thresholds and reducing growth rates. MAFIA calculations of short-range and long-range wakefields have allowed computations of growth rates and thresholds, which are presented here. Resistive wall instability dominates long-range effects, and requires a broadband feedback system to control coupled-bunch motion. Growth rates are within the range addressable by current feedback system technologies. Single-bunch instability thresholds are safely above nominal operating current.
\end{abstract}

\section{DAMPING RINGS DESIGN}

Table 1: Parameters for the Main Damping Ring and Positron Pre-Damping Ring.

\begin{tabular}{|l|c|c|}
\hline & MDR & PPDR \\
\hline Circumference $(\mathrm{m})$ & 299.792 & 218.336 \\
\hline Max. Current $(\mathrm{A})$ & 0.8 & 0.75 \\
\hline Bunch trains $\mathrm{x}$ & $3 \times 190 \times 1.4$ & $2 \times 190 \times 1.4$ \\
Bunches per train $\times$ & or & or \\
Bunch separation & $3 \times 95 \times 2.8$ & $2 \times 95 \times 2.8$ \\
$(\mathrm{~ns})$ & & \\
\hline$v_{\mathrm{x}}, v_{\mathrm{y}}, v_{\mathrm{s}}$ & $27.26,11.14$, & $8.91,7.24$, \\
\hline$\sigma_{\varepsilon}(\%), \sigma_{\mathrm{z}}(\mathrm{mm})$ & 0.0035 & 0.031 \\
\hline$\tau_{\mathrm{x}}, \tau_{\mathrm{y}}, \tau_{\varepsilon}(\mathrm{ms})$ & $4.85,5.09$, & $0.09,7.2$ \\
\hline$\alpha_{\mathrm{p}}$ & 2.61 & $4.24,5.13$, \\
\hline $\mathrm{V}_{\mathrm{RF}}(\mathrm{MV})$ & $2.95 \times 10^{-4}$ & $7.05 \times 10^{-3}$ \\
\hline Frequency $(\mathrm{MHz})$ & 1.07 & 3.4 \\
\hline
\end{tabular}

The purpose of the damping rings is to provide low emittance electron and positron bunch trains to the NLC linacs, at a rate of $120 \mathrm{~Hz}$. The main damping rings operate at $1.98 \mathrm{GeV}$ and may store up to $0.8 \mathrm{amp}$ in 3 trains of 95 bunches each, have bunch lengths of less than $4 \mathrm{~mm}$, and normalized extracted beam emittances $\gamma \varepsilon_{x} \leq 3$ $\mu \mathrm{m}$-rad and $\gamma \varepsilon y \leq 0.03 \mu \mathrm{m}$-rad. The number of particles per bunch is $0.75 \times 10^{10}$ for $1.4 \mathrm{~ns}$ spacing, and $1.5 \times 10^{10}$ for $2.8 \mathrm{~ns}$ spacing. The optical designs are based on a theoretical minimum emittance (TME) lattice, and major parameters are shown in Table $1[1,2]$.

\footnotetext{
* Work supported by the U.S. Department of Energy under Contract Nos. DE-AC03-76SF00515 (SLAC) and DE-AC0376SF00098 (LBNL) †jncorlett@lbl.gov
}

Vacuum chambers are made of aluminum, with antechambers through the arcs to allow adsorption of synchrotron radiation on discrete photon stops electromagnetically isolated from the beam. Beam-pipe apertures are $32 \mathrm{~mm}$ diameter in the arcs and injection/extraction straights, and $16 \mathrm{~mm}$ diameter through the damping wiggler straight. The RF cavities are higher-order-mode damped, with apertures of $64 \mathrm{~mm}$, and the beampipe diameter is increased to $64 \mathrm{~mm}$ through the $\mathrm{RF}$ section [3]. Blends are used to mate sections of different cross-section, using tapers of at least 10:1 aspect ratio. All components are designed with minimizing impedance as a design constraint. Stability of the extracted beam is critical to ensure effective bunch compression and luminosity at the interaction point.

\section{SHORT-RANGE WAKEFIELDS}

3-D MAFIA time-domain models have been used to compute the short-range wakefield for main damping rings beamline components. Major components modeled are

- RF cavities

- antechamber slots

- beam position monitors

- bellows masks

- injection kickers

- feedback kickers

The resistive wall impedance has been calculated analytically.

Wakefields were calculated at the nominal bunch length to determine the impedance budget. A shorter bunch length was used to obtain a Green's function wakefield for beam instability studies. Both longitudinal and transverse wakes have been calculated for the components listed.

While detailed designs are not yet developed for many components, the models used here are based on experience with other rings (such as the ALS at Berkeley, and the PEP-II B-factory), and are believed to represent a realistic set of components.

\subsection{RF cavities}

In order to reduce transverse mode impedance, the cavities have a larger bore than the straight section beampipe. Tapers are located at the ends of the RF section, encompassing the three cavities in the main damping rings. The contribution of the tapers and cavities to the short-range wake has been determined separately, ignoring cross-talk between taper and cavities. The cavities each contribute a loss factor $0.970 \mathrm{VpC}^{-1}$, and the taper pair $0.067 \mathrm{VpC}^{-1}$. The taper impedance is inductive, $0.41 \mathrm{nH}$. To improve accuracy of the taper calculation, the 
mesh density was increased until little change was found with further density increases.

\subsection{Antechamber slot}

The arc vacuum vessel antechamber is isolated from the beam chamber by a slot with aspect ratio height to depth at least 2:1. The impedance of such slots is dominated by the ends, where the slot is gradually opened up from the vacuum chamber wall. We have taken the slot height as $10 \mathrm{~mm}$, depth $20 \mathrm{~mm}$. The resulting wakefield is inductive, and weak. Here we have assumed multiple slots, however the vacuum chamber design will strive to provide continuous slot, reducing the impedance estimate.

\section{$2.3 B P M^{\prime} s$}

Our model uses four PEP-II type button BPM's, with button diameter $8 \mathrm{~mm}$. Due to the large number of these components, their contribution to the impedance budget is significant. We have not addressed the narrow-band impedance of these devices, although it is known that circumferential modes around the button may produce a relatively large resonant impedance at high frequencies.

\subsection{Bellows masks}

Bellows will be shielded by internal structures that carry the beam image currents with minimum perturbation. Such structures are generally not robust in accommodating heat load from synchrotron radiation, and the shield must be masked from the radiation fan. The masks modeled consist of small $(2 \mathrm{~mm})$ indentations in the upstream beampipe, tapered on both sides.

\subsection{Injection and extraction kickers}

A low-impedance kicker design is being pursued for the NLC damping rings. Preliminary studies suggest a DELTA-style kicker will provide the necessary beam deflection with a lower impedance than conventional, especially ferrite-loaded, kickers [4]. In this design, the kicker is in a flat aspect ratio vessel, and the majority of the image currents are carried undisturbed along the top and bottom of the chamber. Current-carrying strips are located to the sides of the chamber.

\subsection{Transverse feedback kickers}

A transverse feedback system will be necessary to control coupled-bunch motion arising from the resistive wall impedance, and perhaps two-beam instabilities. The kickers are simple striplines, one in each plane.

\subsection{Longitudinal resistive wall}

For short bunches and small vacuum chambers, as in the NCL damping rings, the contribution of the longitudinal resistive wall may be significant.

\section{RESONANT IMPEDANCE}

The long-range impedance is expected to be dominated by RF cavity HOM's [3]. The impedance spectrum is shown in figure 2 .
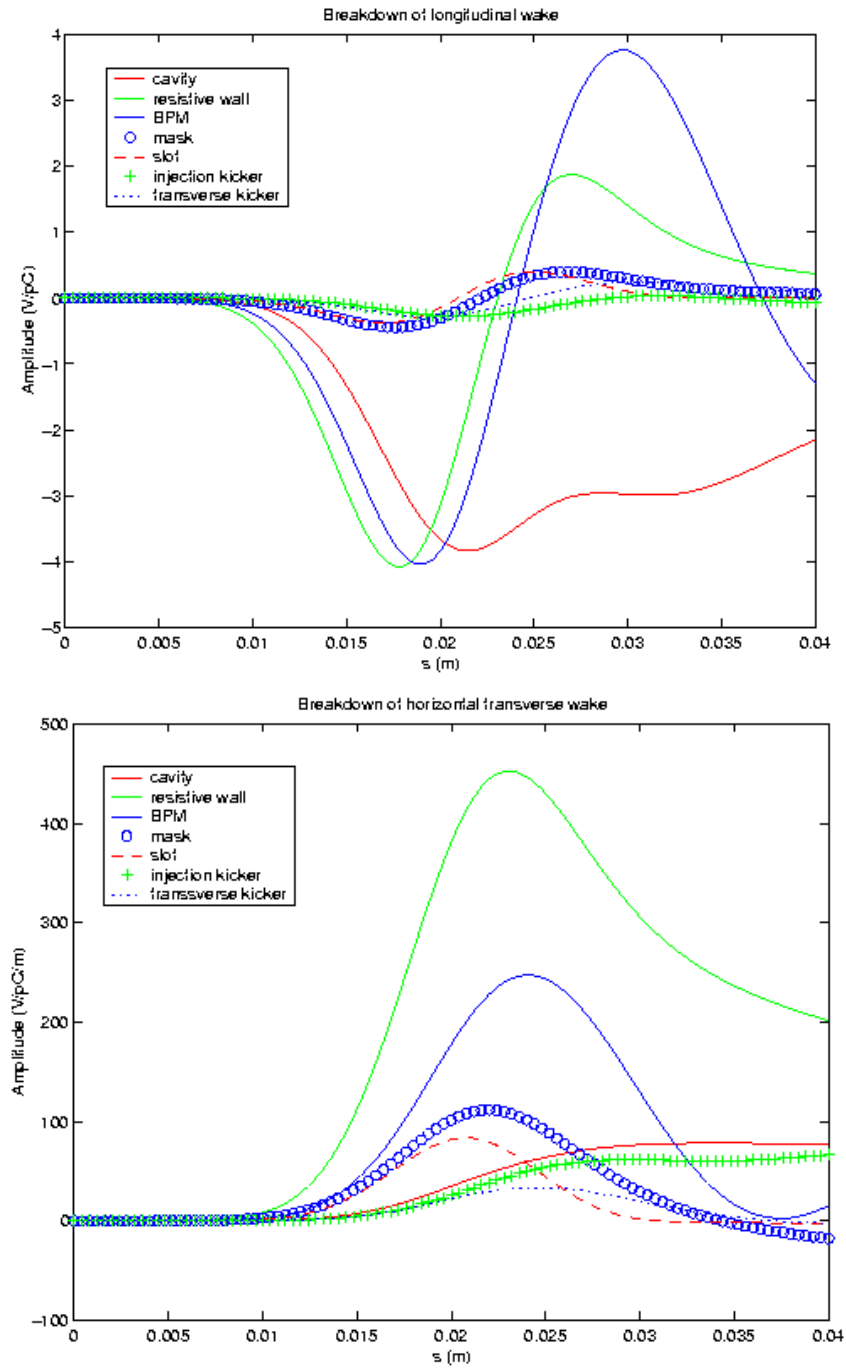

Figure 1. Short-range wakefields, longitudinal (top), horizontal (bottom).

Table 2: Longitudinal broadband impedance budget.

\begin{tabular}{|c|c|c|c|}
\hline Component & Quantity & $\begin{array}{c}\text { Loss } \\
\text { factor } \\
\left(\mathrm{VpC}^{-1}\right)\end{array}$ & $\begin{array}{c}\text { Inductance } \\
(\mathrm{nH})\end{array}$ \\
\hline RF cavity & 3 & 2.976 & 0.41 \\
\hline BPM & 159 & 2.226 & 2.97 \\
\hline Slot & 102 & 0.068 & 0.28 \\
\hline Mask & 102 & 0.168 & 0.33 \\
\hline Kicker & 2 & 0.192 & 0.21 \\
\hline $\begin{array}{c}\text { Feedback } \\
\text { kicker }\end{array}$ & 2 & 0.171 & 0.21 \\
\hline Resistive wall & & 1.867 & \\
\hline TOTAL & & 7.67 & 4.40 \\
\hline
\end{tabular}

\section{INSTABILITY STUDIES}

\subsection{Single-bunch}

The calculated short-range longitudinal wakefield was used in a Fokker-Planck simulation code to study 
longitudinal single-bunch stability. Signs of instability begin to occur at approximately $20 \mathrm{~mA}$ per bunch, a factor of 8 greater than the charge for which the rings are being designed. For the case of maximum charge $1.5 \times 10^{10}$ particles per bunch, we find bunch lengthening of about $14 \%$. Figure 3 shows results of simulations, showing instability at bunch current of $20 \mathrm{~mA}$.

Table 3: Transverse broadband impedance budget.

\begin{tabular}{|c|c|c|c|}
\hline Component & Quantity & $\begin{array}{c}\mathrm{H}^{\mathrm{kick} \text { factor }} \\
\left(\mathrm{VpC}^{-1} \mathrm{~m}^{-1}\right)\end{array}$ & $\begin{array}{c}\mathrm{V} \text { kick factor } \\
\left(\mathrm{VpC}^{-1} \mathrm{~m}^{-1}\right)\end{array}$ \\
\hline RF cavity & 3 & 36.08 & 36.08 \\
\hline BPM & 159 & 146.76 & 146.76 \\
\hline Slot & 102 & 56.34 & 0.13 \\
\hline Mask & 102 & 77.01 & 1.22 \\
\hline Kicker & 2 & 25.4 & 2.86 \\
\hline $\begin{array}{c}\text { Feedback } \\
\text { kicker }\end{array}$ & 2 & 19.05 & 0.40 \\
\hline $\begin{array}{c}\text { Resistive } \\
\text { wall }\end{array}$ & & 320.64 & 320.64 \\
\hline TOTAL & & 681.28 & 508.10 \\
\hline
\end{tabular}
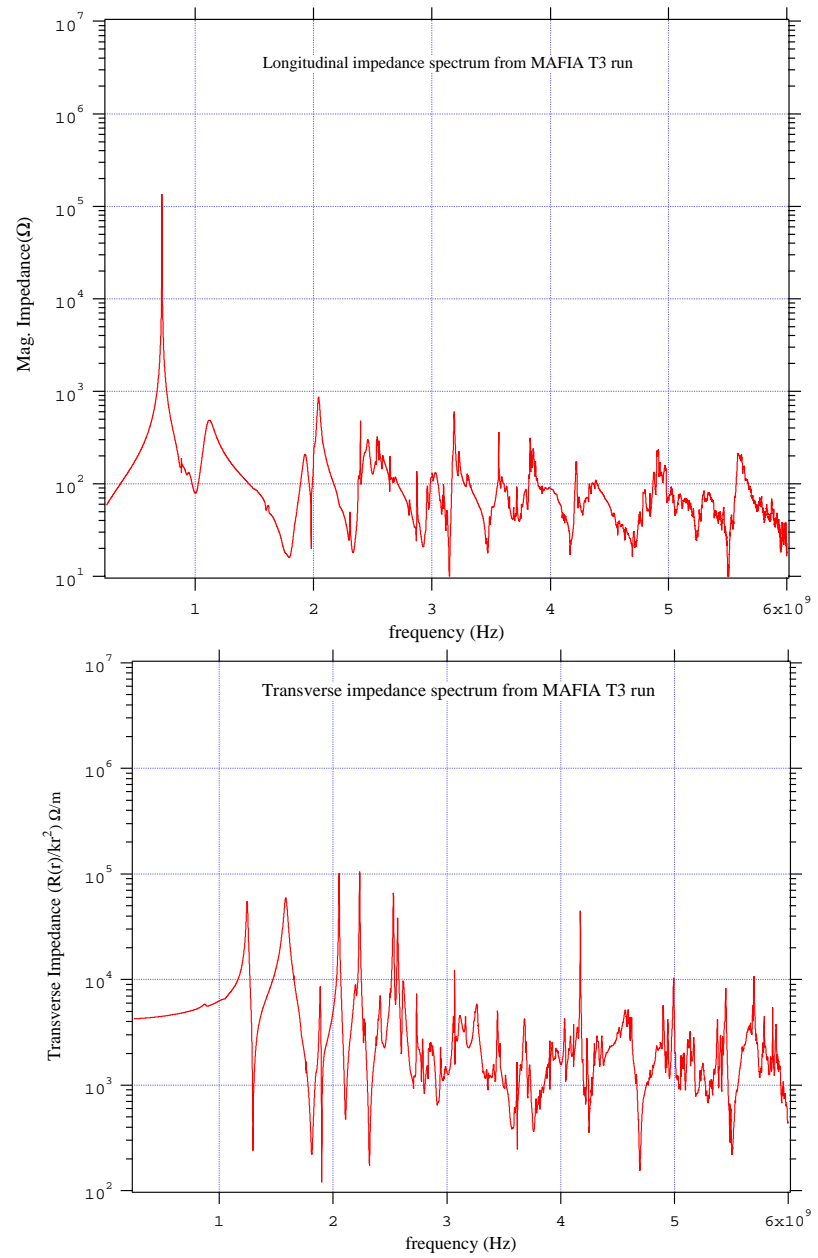

Figure 2. Long-range cavity wakefields, longitudinal (top), transverse (bottom).

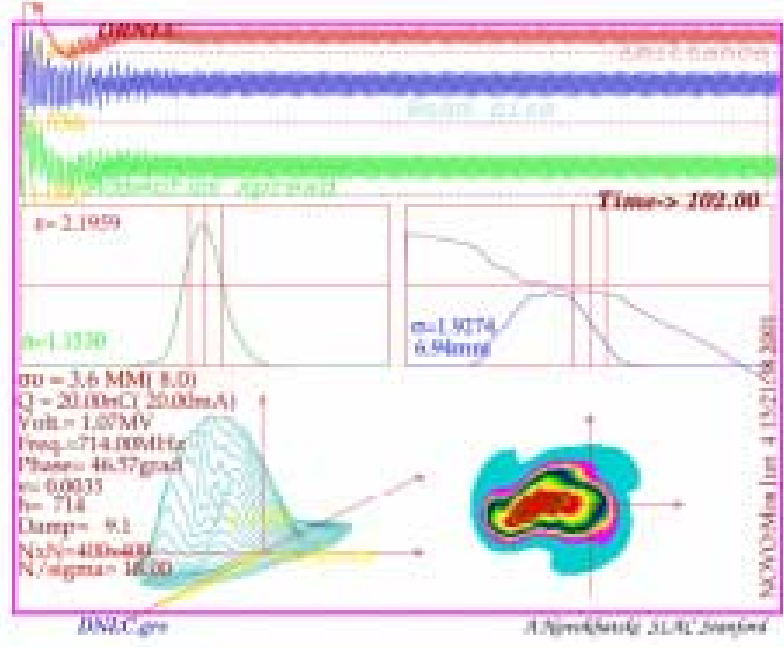

Figure 3. Simulation results for longitudinal single-bunch motion with calculated wakefields.

\subsection{Multi-bunch}

Longitudinal and transverse coupled-bunch growth rates have been estimated from the cavity HOM's and resistive wall impedance. Growth rates are generally faster for the $2.4 \mathrm{~ns}$ bunch spacing case. Longitudinal growth rates are generally below radiation damping rates, apart from two modes driven by the detuned cavity fundamental mode. These must be addressed with a feedback system - possibly around the RF station, as on the PEP-II B-factory.

Transverse coupled-bunch motion is dominated by the effects of the resistive wall. Many modes have growth rates above the radiation damping rate, and a broadband, bunch-by-bunch feedback system will be necessary to control this motion.

\subsection{Electron cloud}

An initial study of the formation of an electron cloud in the positron ring has assumed only a circular beampipe without antechamber. The electron density is found to be sensitive to photoemission, but not to the secondary emission yield of the vacuum chamber walls. By displacing a bunch in the simulation we estimate the wakefield and from that determine the growth rates of coupled bunch modes. The fastest mode growth time is approximately $100 \mu \mathrm{s}$, and may be damped by a broadband feedback system.

\section{REFERENCES}

[1] J. Corlett et al, "The Next Linear Collider Damping Ring Complex", this conference.

[2] A. Wolski and J. Corlett, " The Next Linear Collider Damping Ring Lattices", this conference.

[3] R.A. Rimmer et al, "An RF cavity for the NLC damping rings", this Conference.

[4] G. Blokesch, et al, "A Slotted Pipe Kicker for High Current Storage Rings", Nucl.Instrum.Meth. A338:151155,1994 . 